\section{Insuficiencia renal aguda secundaria a rabdomiolisis como manifestación de infección por SARS-CoV-2}

\author{
JAVIER PÉREZa, SEBASTIÁN SÁNCHEZ ${ }^{\text {a }}$

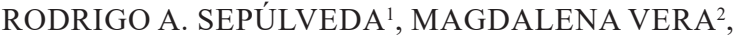 \\ PABLO MERY ${ }^{3}$, BERNARDITA GARAYAR ${ }^{4}$, \\ ROBERTO JALIL ${ }^{1}$
}

\section{Acute kidney injury caused by} rhabdomyolysis as a SARS-CoV-2 infection manifestation. Report of one case

SARS-CoV-2 infection has a wide spectrum of clinical manifestations secondary to the impairment of different organs, including kidney. Rhabdomyolysis is produced by disintegration of striated muscle and the liberation of its contents to the extracellular fluid and bloodstream. This may produce hydro electrolytic disorders and acute kidney injury. We report a 35-year-old female with a history of SARS-CoV-2 infection who was hospitalized because of respiratory failure and developed renal failure. The etiologic study showed elevated total creatine kinase levels and a magnetic resonance imaging confirmed rhabdomyolysis. The patient required supportive treatment with vasoactive drugs, mechanic ventilation and kidney replacement therapy. She had a favorable evolution with resolution of respiratory failure and improvement of kidney function.

(Rev Med Chile 2021; 149: 641-647)

Key words: Acidosis; Acute Kidney Injury; COVID-19; Rhabdomyolysis.
'Departamento de Nefrología, Facultad de Medicina, Pontificia Universidad Católica de Chile. Santiago, Chile.

${ }^{2}$ Departamento de Medicina Intensiva, Facultad de Medicina, Pontificia Universidad Católica de Chile. Santiago, Chile.

${ }^{3}$ Departamento de Traumatología, Facultad de Medicina, Pontificia Universidad Católica de Chile. Santiago, Chile.

${ }^{4}$ División de Enfermedades Cardiovasculares, Facultad de Medicina, Pontificia Universidad Católica de Chile. Santiago, Chile. anterno de medicina, Facultad de Medicina, Pontificia Universidad Católica de Chile. Santiago, Chile.

Trabajo no recibió financiamiento. Los autores declaran no tener conflictos de interés.

Recibido el 29 de octubre de 2020, aceptado el 12 de marzo de 2021.

Correspondencia a: Rodrigo Sepúlveda P. Palamara Diagonal Paraguay 362, Departamento de Nefrología. $4^{\circ}$ piso. Santiago, Chile. rrsepulveda@uc.cl
$\mathrm{L}$ a infección por SARS-CoV-2 ha tomado gran relevancia desde su aparición, no solo por su gran impacto con más de 200 países afectados, 10 millones de casos confirmados y 500.000 muertes atribuibles a nivel mundial para el 1 de julio de $2020^{1}$. Sino también, su espectro variable de presentación que va desde casos asintomáticos (4-32\% $)^{2,3}$, aquellos que se presentan típicamente con fiebre, tos seca, disnea, fatiga, mialgias, náuseas/vómitos, diarrea, cefalea, debilidad, rinorrea, anosmia y/o ageusia; y los casos graves que pueden cursar con neumonia, distrés respiratorio, shock distributivo y otras complicaciones con elevada morbimortalidad $^{4}$. Se sabe que alrededor de 14\% se presentarán de forma severa, fundamentalmente con complicaciones respiratorias (principal cau- sa de admisión a unidad de paciente crítico), shock séptico, disfunción hepática, accidente vascular encefálico o encefalitis, complicaciones cardiacas, enfermedad tromboembólica e insuficiencia renal aguda (IRA), entre otros ${ }^{4}$.

La rabdomiolisis es producida por la necrosis muscular y paso del contenido intracelular a la sangre. Se manifiesta principalmente por mialgias (ausentes en 50\%) ${ }^{5}$, mioglobinuria y aumento de la creatina-quinasa total $(\mathrm{CK})^{6}$. La severidad de la enfermedad se asocia a mayores niveles de CK, pudiendo desencadenar trastornos hidroelectrolíticos e IRA.

A continuación se presenta el caso de una paciente que cursa con una IRA secundaria a rabdomiolisis relacionada con SARS-CoV-2. 


\section{Caso clínico}

Mujer de 35 años con antecedentes de sobrepeso, 3 gestaciones y 3 partos vaginales espontáneos sin complicaciones. Sin hábitos tóxicos ni consumo de fármacos. Cursa una infección por SARS-CoV-2 confirmada con PCR, caracterizada por compromiso del estado general, fiebre e historia de polimialgias difusas de predominio en extremidades inferiores, con postración de la paciente 2 días previo a ingreso. Se agrega disnea rápidamente progresiva por lo que es llevada a nuestro servicio de urgencia. A su ingreso destaca hipotensa 67/47 $\mathrm{mm} \mathrm{Hg}$, mal perfundida, en sopor, saturando $75 \%$, polipneica. Se inicia reanimación con fluidos y se conecta a ventilación mecánica invasiva.

El laboratorio de ingreso se muestra en la Tabla 1. Destaca una intensa acidosis con pH 6,94, $\mathrm{HCO}_{3}-10,5 \mathrm{mEq} / \mathrm{L}, \mathrm{PaO}_{2} 189 \mathrm{mmHg}$, con $\mathrm{FiO}_{2}$ $80 \%, \mathrm{PaCO}_{2} 49 \mathrm{~mm} \mathrm{Hg}$, proteína C reactiva 2,16 $\mathrm{mg} / \mathrm{dL}$, lactato $8,7 \mathrm{mmol} / \mathrm{L}$, creatininemia 2,26 $\mathrm{mg} / \mathrm{dL}$, potasio $5,5 \mathrm{mEq} / \mathrm{L}$.

Tabla 1. Exámenes de ingreso

\begin{tabular}{|c|c|c|c|}
\hline Gases arteriales & $\begin{array}{l}\mathrm{PaO}_{2} \\
\mathrm{SaO}_{2} \\
\mathrm{PaCO}_{2} \\
\mathrm{pH} \\
\text { Bicarbonato real } \\
\text { Exceso de base } \\
\text { Lactato arterial }\end{array}$ & $\begin{array}{l}189,0 \mathrm{mmHg} \\
99,9 \% \\
49,0 \mathrm{mmHg} \\
6,94 \\
10,5 \mathrm{mEq} / \mathrm{L} \\
-22,3 \mathrm{mmol} / \mathrm{L} \\
8,7 \mathrm{mmol} / \mathrm{L}\end{array}$ & $\begin{array}{l}(V N: 75,5-85,5) \\
(V N: 90-100) \\
(V N: 35-45) \\
(V N: 7,35-7,45) \\
(V N: 21-27) \\
(V N:-3,0-3,0) \\
(V N: 0,9-1,7)\end{array}$ \\
\hline Bioquímica & $\begin{array}{l}\text { Glicemia } \\
\text { Creatinina } \\
\text { Nitrógeno ureico en sangre } \\
\text { Albúmina } \\
\text { Sodio } \\
\text { Potasio } \\
\text { Cloro } \\
\text { Calcio } \\
\text { Fosfato } \\
\text { Ácido úrico }\end{array}$ & $\begin{array}{l}150 \mathrm{mg} / \mathrm{dL} \\
2,26 \mathrm{mg} / \mathrm{dL} \\
50 \mathrm{mg} / \mathrm{dL} \\
3,1 \mathrm{gr} / \mathrm{dL} \\
134 \mathrm{mEq} / \mathrm{L} \\
5,5 \mathrm{mEg} / \mathrm{L} \\
100 \mathrm{mEq} / \mathrm{L} \\
6,4 \mathrm{mg} / \mathrm{dL} \\
8,53 \mathrm{mg} / \mathrm{dL} \\
16,7 \mathrm{mg} / \mathrm{dL}\end{array}$ & $\begin{array}{l}(\mathrm{VN}: 70-99) \\
(\mathrm{VN}: 0,5-0,9) \\
(\mathrm{VN}: 8-25) \\
(\mathrm{VN}: 3,5-5) \\
(\mathrm{VN}: 135-145) \\
(\mathrm{VN}: 3,5-5) \\
(\mathrm{VN}: 100-108) \\
(\mathrm{VN}: 8,5-10,5) \\
(\mathrm{VN}: 2,6-4,5) \\
(\mathrm{VN}: 2,3-6,6)\end{array}$ \\
\hline Examen de orina* & $\begin{array}{l}\mathrm{pH} \\
\text { Eritrocitos } \\
\text { Leucocitos } \\
\text { Células descamativas } \\
\text { Bacterias } \\
\text { Cristales }\end{array}$ & $\begin{array}{l}5 \\
++++ \\
6 \text { por campo d } \\
+ \\
+ \\
\text { Urato amorfo+ }\end{array}$ & e mayor aumento \\
\hline $\begin{array}{l}\text { Parámetros } \\
\text { severidad } \\
\text { COVID-19 }\end{array}$ & $\begin{array}{l}\text { - Proteína C reactiva } \\
\text { - Lactato deshidrogenasa } \\
\text { - Ferritina } \\
\text { - Dímero D } \\
\text { - Interleukina-6 } \\
\text { - Recuento absoluto linfocitos }\end{array}$ & $\begin{array}{l}19,32 \mathrm{mg} / \mathrm{dL} \\
1.701 \mathrm{U} / \mathrm{L} \\
6.986 \mathrm{ng} / \mathrm{mL} \\
3.028 \mathrm{ng} / \mathrm{mL} \\
132,9 \mathrm{pg} / \mathrm{mL} \\
1,94 \times 10^{3} / \mu \mathrm{L}\end{array}$ & $\begin{array}{l}(\mathrm{VN}:<0,5) \\
(\mathrm{VN}: 135-214) \\
(\mathrm{VN}: 13-150) \\
(\mathrm{VN}:<=500) \\
(\mathrm{VN}:<=7) \\
(\mathrm{VN}: 1,0-4,8)\end{array}$ \\
\hline $\begin{array}{l}\text { Estudio } \\
\text { microbiológico }\end{array}$ & $\begin{array}{l}\text { - Hemocultivos I y II } \\
\text { - Urocultivo } \\
\text { - Antígeno urinario S. pneumoniae } \\
\text { - Antígeno urinario Legionella } \\
\text { - Panel molecular respiratorio }{ }^{\dagger}\end{array}$ & \multicolumn{2}{|c|}{$\begin{array}{l}\text { (+) Streptococcus hominis ( } 21,2 \text { y } 18,1 \mathrm{~h}) \text {; } \\
\text { (+) Staphylococcus epidermidis ( } 35 \text { y 17,6 h) } \\
\text { negativo } \\
\text { negativo } \\
\text { negativo } \\
\text { negativo }\end{array}$} \\
\hline
\end{tabular}

Abreviaciones: VN: valor normal. *Examen de sedimento urinario obtenido posterior a instalación de catéter urinario permanente. 'Examen (mediante reacción en cadena de la polimerasa, múltiple) incluye estudio de: Adenovirus, Coronavirus 229E, Coronavirus HKU1, Coronavirus NL63, Coronavirus OC43, Metapneumovirus, Rinovirus/Enterovirus, Influenza A y B, Parainfluenza 1, 2, 3 y 4, Virus Sincicial Respiratorio, Bordetella pertussis, Bordetella parapertussis, Chlamydophila pneumoniae, Mycoplasma pneumoniae. 
Ingresa grave a Unidad de Cuidados Intensivos, con alto requerimiento de drogas vasoactivas. Inicialmente se interpreta como una falla respiratoria por COVID-19 sin poder descartar un compromiso séptico secundario, dada la intensidad del shock; por lo que se amplió estudio con imágenes, hemocultivos y se agrega antibióticos. La tomografía computada de tórax sin contraste, muestra una extensa neumonía multifocal compatible con COVID-19, sin otros hallazgos. En el examen físico destaca edema y empastamiento de ambos muslos, se realiza eco doppler venoso de extremidades inferiores que descarta trombosis venosa. Dado cuadro de shock séptico severo, se solicita resonancia magnética de ambas extremidades inferiores, sin contraste, para descartar fascitis necrotizante. Ésta objetiva signos de rabdomiolisis y miositis difusa, simétrica, sobre todo en extremidades inferiores (Figura 1). Se controla CK total la cual resulta muy elevada $(61.290 \mathrm{U} / \mathrm{L})$. Se plantea como causas de la acidosis: la hipoperfusión sistémica (acidosis láctica), rabdomiolisis, falla renal

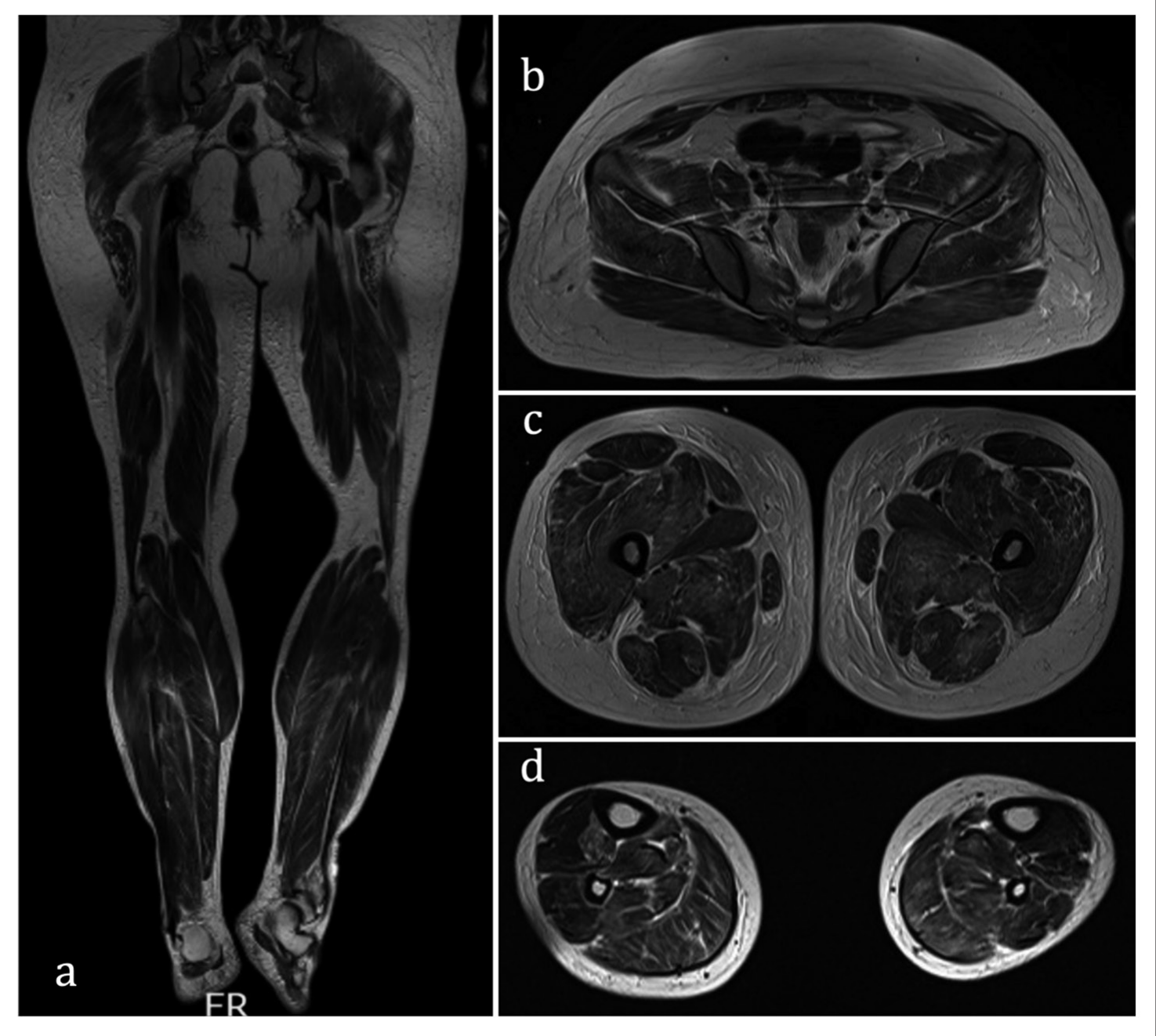

Figura 1. Resonancia magnética de ambas extremidades inferiores sin gadolinio endovenoso. Imágenes correspondientes a cortes coronales (a) y axiales a nivel de pelvis (b), tercio medio de muslos (c) y tercio medio de piernas (d). Se observan hallazgos compatibles con rabdomiolisis difusa, manifestados por aumento de señal secundario a cambios inflamatorios y edema difuso de vientres musculares de ambos muslos y piernas, con compromiso relativamente simétrico de musculatura glútea, iliopsoas, aductores, cuádriceps y músculos de los diferentes compartimientos de las piernas. Presencia de contenido hipertenso en vientres de músculos pectíneo, vastos intermedios y laterales de ambos muslos, compatible con hemorragia intramuscular. 


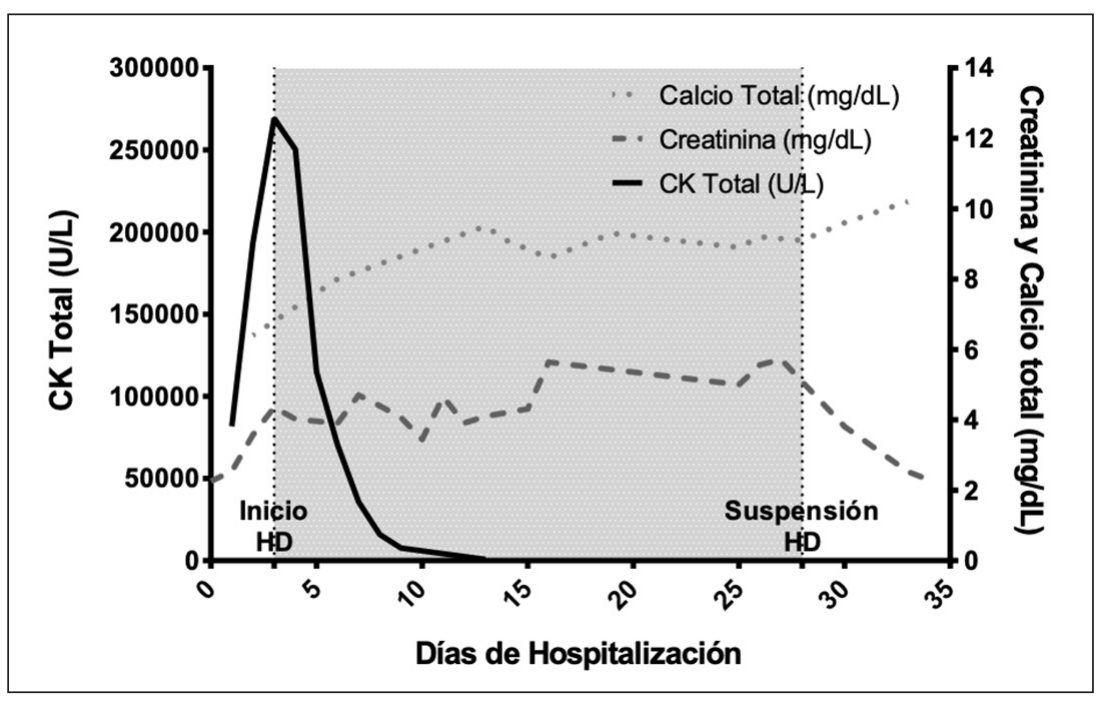

Figura 2. Evolución parámetros durante hospitalización. Se objetiva una rabdomiolisis con intenso aumento de CK Total que normaliza a los pocos días. La zona gris corresponde al período de tiempo donde requirió terapia de reemplazo renal. Al mes de evolución recupera función renal y se estabiliza la calcemia en un rango normal-alto. e insuficiencia respiratoria. Se inicia manejo con bicarbonato de sodio IV y fluidos, pero evoluciona con insuficiencia renal aguda progresiva pese a restaurar alteraciones hemodinámicas. En los días posteriores, la CK llega hasta 269.328 U/L. En contexto de insuficiencia renal progresiva, hiperkalemia y anuria se inicia terapia de reemplazo renal al tercer día de hospitalización.

Como etiologías de la falla renal, se presume una injuria tubular aguda asociada a la rabdomiolisis, el estado de hipoperfusión renal, más una respuesta inflamatoria sistémica e infección por SARS-CoV-2.

Se realiza estudio etiológico de miositis con pruebas inmunológicas: ANA (+) 1/80 nuclear moteado, ANCA (-), niveles de complemento C3 y C4 normales, Perfil ENA (-), panel de miopatías inflamatorias (-).

Del estudio infectológico se obtiene hemocultivos tardíamente positivos para S. Hominis y S. Viridans (con sospecha de contaminación), se escala cobertura a Imipenem más Vancomicina. El resto del estudio microbiológico fue negativo. Se realizó ecocardiograma transtorácico que no muestra vegetaciones. Completa empíricamente 7 días de Imipenem y 10 días de Vancomicina. No recibió corticoides ni otra terapia inmunosupresora por dudosa infección bacteriana.

Evoluciona favorablemente con mejoría en parámetros hemodinámicos y ventilatorios. Re- quirió 11 días de drogas vasoactivas y 12 días de ventilación mecánica invasiva.

En lo nefrológico, con la terapia de reemplazo renal se normalizan alteraciones del medio interno. Trece días después de su ingreso, comienza a presentar débito urinario progresivo hasta 1500 $\mathrm{mL} / \mathrm{d}$. Finalmente aparece función renal depurativa y luego de 30 días de evolución con terapia de reemplazo renal, ésta logra ser discontinuada (Figura 2).

Con remisión espontánea de la rabdomiolisis, neumonía grave por SARS-CoV-2 en recuperación, rehabilitación satisfactoria pudiendo realimentarse y deambular sin ayuda, y además, con un aclaramiento de creatinina de $28 \mathrm{~mL} / \mathrm{min}$; es dada de alta para continuar su rehabilitación y manejo ambulatorio. Previo al egreso hospitalario la paciente autoriza, mediante un consentimiento informado, el reporte de su caso clínico.

\section{Discusión}

La pandemia producida por SARS-CoV-2 ha significado un gran desafío para la humanidad. Además de todos los problemas médicos, sociales y económicos que genera; debemos resaltar que es una enfermedad con una presentación clínica extremadamente variable. Lo anterior se hace más evidente en los cuadros graves, provocando 
múltiples formas de daño orgánico con diagnóstico y enfrentamiento complejo, además de un pronóstico aún incierto.

La rabdomiolisis ya ha sido reportada como complicación producto de infecciones graves por SARS-CoV-2 ${ }^{7-10}$. La asociación entre un cuadro de COVID-19 severo más la presencia de mialgias, especialmente en extremidades inferiores, han sido factores relacionados con el desarrollo de rabdomiolisis; ambos presentes en nuestra paciente ${ }^{7}$.

El músculo es uno de los tejidos que expresa en la superficie de su membrana plasmática la enzima convertidora de angiotensina tipo II (ECA2), necesaria para la infección por SARS-CoV-2 ${ }^{11}$. Esto podría explicar la alta prevalencia de mialgias en los cuadros de COVID-19 ${ }^{12}$. En pacientes infectados solo $1 \%$ de las muestras sanguíneas presentan virus detectable ${ }^{13}$, pero esta sería la vía de infección para los parénquimas fuera del pulmón ${ }^{14}$. Ciertas miopatías genéticas o el ejercicio, aumentan la expresión de ECA2 muscular ${ }^{15,16}$. Desconocemos si nuestra paciente presentaba alguna de las condiciones explicitadas pero podrían crear vulnerabilidad al desarrollo de rabdomiolisis por SARS-CoV-2.

La destrucción muscular masiva provoca secuestro de líquido ${ }^{17,18}$, esto podría explicar el intenso compromiso hemodinámico de la paciente. A esto se agrega que la acidemia grave $(\mathrm{pH}<7,2)$ altera severamente la función cardiovascular e induce resistencia a la actividad adrenérgica ${ }^{19}$.

La acidosis en rabdomiolisis tiene etiología multifactorial. Inicialmente habrá un evento miotóxico capaz de dañar el miocito. Luego, el Ca++ ingresa a la célula induciendo una contracción muscular mantenida. El ATP es necesario para inducir relajación, situación que provoca una intensa actividad metabólica en la célula, generando ácidos orgánicos. Con la destrucción celular saldrán a la circulación sistémica: ácidos orgánicos, $\mathrm{CO}_{2}$, ácido úrico y ácidos provenientes del catabolismo proteico (ácido fosfórico y ácido sulfúrico). También se libera mioglobina, que provoca daño renal ${ }^{17,20}$. Posteriormente, el secuestro de líquido con hipoperfusión sistémica ocasionará más falla renal y acidosis láctica ${ }^{18}$. La liberación masiva de ácidos, falla renal y acidosis láctica pueden haber configurado, en nuestra paciente, la acidosis metabólica grave con la cual se presentó.

El síndrome edematoso puede ser intensificado por el secuestro de líquido en el músculo.
Se describe tendencia a hipocalcemia inicial (por secuestro) y luego hipercalcemia (por liberación desde el músculo $)^{17}$. Es posible que esta última alteración no haya sido evidente por la instauración de terapia de reemplazo renal con $\mathrm{Ca}++$ bajo en el líquido de diálisis.

La falla renal de nuestra paciente tiene un origen multifactorial. El $75 \%$ de los pacientes con neumonía COVID-19 presenta anomalías urinarias (como hematuria y/o proteinuria) $)^{21}$, y $25 \%$ de los casos graves tiene IRA ${ }^{22}$. Nuestra paciente evolucionó precozmente a la anuria, pero en el sedimento urinario de ingreso se observan abundantes hematíes (Tabla 1); pudiendo explicarse por una injuria renal por COVID-19. El virus posee un tropismo especial por el riñón. Los túbulos, podocitos y endotelio renal expresan intensamente ECA2 ${ }^{23}$. El virus induce daño tubular directo severo mediante activación del complemento y reclutamiento de macrófagos ${ }^{22,23}$. A nivel capilar glomerular predomina el daño endotelial y agregación eritrocitaria; sin existir agregados plaquetarios, fibrinoides, vasculitis, ni nefritis intersticial ${ }^{24}$. A la injuria renal directa mediada por el virus, se suman otras formas de daño renal. Existe un estado protrombótico con afectación multiorgánica, $71 \%$ de los pacientes fallecidos por COVID-19 presentan coagulación intravascular diseminada ${ }^{25}$. La respuesta inflamatoria sistémica, el daño pulmonar inducido por ventilación mecánica, y también, la tormenta de citoquinas; generan mediadores que inducen daño tubular directo y alteran el flujo sanguíneo intra-renal ${ }^{26-28}$. Por otro lado, múltiples factores provocarán isquemia renal, como el shock distributivo y disfunción miocárdica asociadas a la sepsis $^{29}$, la hipovolemia producto de secuestro de líquido en el músculo, y también, la ventilación mecánica con presión positiva elevada al final de espiración. La disminución del retorno venoso e incremento de presión venosa central, afectan el gradiente de perfusión renal. Finalmente se agrega el uso de nefrotóxicos y el daño inducido por rabdomiolisis. La destrucción muscular libera mediadores vasoconstrictores a la circulación. Por otro lado, la mioglobina induce estrés oxidativo, y así, disfunción endotelial. La contracción del intravascular por secuestro de líquido en el músculo, sumado a los mediadores vasoconstrictores y la disfunción endotelial; producen una severa disminución del flujo sanguíneo renal. Luego, el 
exceso de mioglobina filtra por el glomérulo, concentrándose en los túbulos generando citotoxidad directa y obstrucción tubular al interactuar con uromodulina ${ }^{17,20}$.

Reconocer una rabdomiolisis en un paciente con COVID-19 severo es extremadamente importante, ya que tiene implicancias en el tratamiento y pronóstico. Al igual que en nuestra paciente, puede presentar gran compromiso hemodinámico e intensa falla renal. Del mismo modo, instaurando una volemización abundante, corrigiendo la acidosis, evitando nefrotóxicos y entregando las medidas adecuadas de soporte; quizás puede ser posible evitar una falla renal severa, y así, la necesidad de terapia de reemplazo renal.

Destacamos que nuestra paciente recuperó función renal pudiendo discontinuar la hemodiálisis. Esto podría entregar un antecedente sobre un pronóstico favorable, aun cuando haya existido una infección grave por SARS-CoV-2 y una rabdomiolisis severa. Será necesario procurar medidas que favorezcan la regeneración del parénquima renal en espera de su recuperación.

\section{Referencias}

1. World Health Organization. Situation report COVID-19. Disponible en: https://www.who.int/docs/ default-source/coronaviruse/situation-reports/20200701 -covid-19-sitrep-163.pdf?sfvrsn=c202f05b_2 [Consultado el 17 de julio de 2020].

2. Byambasuren O, Cardona M, Bell K, Clark J, McLaws M, Glasziou P. Estimating the extent of asymptomatic COVID-19 and its potential for community transmission: systematic review and meta-analysis. medRxiv 2020; 5 (10): 20097543.

3. Tabata S, Imai K, Kawano S, Ikeda M, Kodama T, Miyoshi $\mathrm{K}$ et al. Clinical characteristics of COVID-19 in 104 people with SARS-CoV-2 infection on the Diamond Princess cruise ship: a retrospective analysis. Lancet Infect Dis 2020; 20 (9): 1043-50.

4. Wiersinga WJ, Rhodes A, Cheng AC, Peacock SJ, Prescott HC. Pathophysiology, Transmission, Diagnosis, and Treatment of Coronavirus Disease 2019 (COVID-19): A Review. JAMA 2020; 324 (8): 782-93.

5. Gabow PA, Kaehny WD, Kelleher SP. The spectrum of rhabdomyolysis. Medicine (Baltimore) 1982; 61: 141.

6. Giannoglou GD, Chatzizisis YS, Misirli G. The syndrome of rhabdomyolysis: Pathophysiology and diagnosis. Eur J Intern Med 2007; 18: 90.
7. Jin M, Tong Q. Rhabdomyolysis as Potential Late Complication Associated with COVID-19. Emerg Infect Dis 2020;26 (7): 1618-20.

8. Suwanwongse K, Shabarek N. Rhabdomyolysis as a Presentation of 2019 Novel Coronavirus Disease. Cureus 2020;12 (4): e7561.

9. Mukherjee A, Ghosh R, Aftab G. Rhabdomyolysis in a Patient With Coronavirus Disease 2019. Cureus 2020; 12 (7): e8956.

10. Borku Uysal B, Ikitimur H, Yavuzer S, Islamoglu MS, Cengiz M. Case Report: A COVID-19 Patient Presenting with Mild Rhabdomyolysis. Am J Trop Med Hyg 2020; 103 (2): 847-850.

11. Li MY, Li L, Zhang Y, Wang XS. Expression of the SARS-CoV-2 cell receptor gene ACE2 in a wide variety of human tissues. Infect Dis Poverty 2020;9 (1): 45.

12. Huang C, Wang Y, Li X, Ren L, Zhao J, Hu Y et al. Clinical features of patients infected with 2019 novel coronavirus in Wuhan, China. Lancet 2020; 395 (10223): 497-506.

13. Wang W, Xu Y, Gao R, Lu R, Han K, Wu G, et al. Detection of SARS-CoV-2 in Different Types of Clinical Specimens. JAMA 2020;323 (18): 1843-4.

14. Zhang Y, Geng X, Tan Y, Li Q, Xu C, Xu J, et al. New understanding of the damage of SARS-CoV-2 infection outside the respiratory system. Biomed Pharmacother 2020; 127: 110195.

15. Riquelme C, Acuña MJ, Torrejón J, Rebolledo D, Cabrera $\mathrm{D}$, Santos RA, et al. ACE2 is augmented in dystrophic skeletal muscle and plays a role in decreasing associated fibrosis. PLoS One 2014; 9 (4): e93449.

16. Echeverría-Rodríguez O, Gallardo-Ortíz IA, Del Valle-Mondragón L, Villalobos-Molina R. Angiotensin-(1-7) Participates in Enhanced Skeletal Muscle Insulin Sensitivity After a Bout of Exercise. J Endocr Soc 2020; 4 (2): bvaa007.

17. Bosch X, Poch E, Grau JM. Rhabdomyolysis and acute kidney injury. N Engl J Med 2009;361 (1): 62-72.

18. Torres PA, Helmstetter JA, Kaye AM, Kaye AD. Rhabdomyolysis: pathogenesis, diagnosis, and treatment. Ochsner J 2015; 15 (1): 58-69.

19. Schotola H, Toischer K, Popov AF, Renner A, Schmitto JD, Gummert J et al. Mild metabolic acidosis impairs the $\beta$-adrenergic response in isolated human failing myocardium. Crit Care 2012; 16 (4): R153.

20. McCarron DA, Elliott WC, Rose JS, Bennett WM. Severe mixed metabolic acidosis secondary to rhabdomyolysis. Am J Med 1979; 67 (5): 905-8.

21. Pei G, Zhang Z, Peng J, Liu L, Zhang C, Yu C, et al. Renal Involvement and Early Prognosis in Patients with 
COVID-19 Pneumonia. J Am Soc Nephrol. 2020; 31 (6): 1157-65.

22. Fanelli V, Fiorentino M, Cantaluppi V, Gesualdo L, Stallone G, Ronco C et al. Acute kidney injury in SARSCoV-2 infected patients. Crit Care 2020; 24 (1): 155.

23. Benedetti C, Waldman M, Zaza G, Riella LV, Cravedi P. COVID-19 and the Kidneys: An Update. Front Med (Lausanne). 2020; 7: 423.

24. Su H, Yang M, Wan C, Yi LX, Tang F, Zhu HY, et al. Renal histopathological analysis of 26 postmortem findings of patients with COVID-19 in China. Kidney Int. 2020; 98 (1): 219-27.

25. Tang N, Bai H, Chen X, Gong J, Li D, Sun Z. Anticoagulant treatment is associated with decreased mortality in severe coronavirus disease 2019 patients with coagu- lopathy. J Thromb Haemost. 2020; 18 (5): 1094-9.

26. Schrier RW, Wang W. Acute renal failure and sepsis. N Engl J Med. 2004; 351 (2): 159-69.

27. Honore PM, Jacobs R, Joannes-Boyau O, De Regt J, Boer W, De Waele E, et al. Septic AKI in ICU patients. diagnosis, pathophysiology, and treatment type, dosing, and timing: a comprehensive review of recent and future developments. Ann Intensive Care. 2011; 1 (1): 32.

28. Hepokoski ML, Malhotra A, Singh P, Crotty Alexander LE. Ventilator-Induced Kidney Injury: Are Novel Biomarkers the Key to Prevention? Nephron. 2018; 140 (2): 90-3.

29. Rudiger A, Singer M. Mechanisms of sepsis-induced cardiac dysfunction. Crit Care Med. 2007; 35 (6): 1599 608. 